\title{
An automatic method for prostate segmentation on 3D MRI scans using local phylogenetic indexes and XGBoost
}

\author{
Giovanni L. F. da Silva ${ }^{1}$, Francisco Y. C. de Oliveira ${ }^{1}$, João O. B. Diniz ${ }^{1}$, \\ Petterson S. Diniz ${ }^{1}$, Darlan B. P. Quintanilha ${ }^{1}$, Aristófanes C. Silva ${ }^{1}$, \\ Anselmo C. de Paiva ${ }^{1}$, Elton A. A. de Cavalcanti ${ }^{2}$ \\ ${ }^{1}$ Federal University of Maranhão - UFMA, Applied Computer Group - NCA \\ Av. dos Portugueses, SN, Bacanga, 65085-580, São Luís, MA, Brazil \\ ${ }^{2}$ University Hospital of the Federal University of Maranhão - HUUFMA \\ R. Barão de Itapary, 227, Centro, 65020-070, São Luís, MA, Brazil \\ \{giovannilucca, ylderlan, joao.bandeira\}@nca.ufma.br, \\ \{petterson.diniz, dquintanilha, ari, paiva\}@nca.ufma.br, \\ eltoncavalcanti@gmail.com
}

\begin{abstract}
The detection, diagnosis, and treatment of prostate cancer depends on the correct determination of the prostate anatomy. In current practice, the prostate segmentation is performed manually by a radiologist, which is extremely time-consuming that demands experience and concentration. Therefore, this paper proposes an automatic method for prostate segmentation on $3 D$ magnetic resonance imaging scans using a superpixel technique, phylogenetic indexes, and an optimized XGBoost algorithm. The proposed method has been evaluated on the Prostate 3T and PROMISE12 databases presenting a dice similarity coefficient of $84.48 \%$ and a volumetric similarity of 95.91\%, demonstrating the high-performance potential of the proposed method.
\end{abstract}

\section{Introduction}

According to the American Cancer Society, 248,530 new cases of prostate cancer are estimated for 2021, with a rate of 34,130 deaths in the United States [ACS 2021]. Except for skin cancer, prostate cancer is the most common cancer among men, being the second leading cause of cancer deaths [Siegel et al. 2019]. Nonetheless, the death rate has decreased by approximately 51\% from 1993 to 2016 due to increased screening. Based on recent data, about $12.5 \%$ of men develop prostate cancer, with $60 \%$ of cases starting at age 65 [INCA 2021].

The detection, diagnosis, and treatment, such as biopsy and radiotherapy procedures, depends on the correct determination of the prostate anatomy. Thereby, several studies [Paiva et al. 2019, Comelli et al. 2021] propose computer-aided methods for prostate segmentation on various medical imaging modalities. The Magnetic Resonance Imaging (MRI) scan is the most convenient modality for cancer detection and staging due to high spatial resolution and excellent soft-tissue contrast compared to Transrectal Ultrasound (TRUS) and Computed Tomography (CT) scans [Ghose et al. 2012]. 
In current practice, the prostate segmentation is performed manually by a radiologist, based on a slice by slice visual inspection, since the MRI scan provides a three-dimensional view [Ghose et al. 2012]. This manual segmentation approach is extremely time-consuming that demands experience, concentration, and is prone to variability among different radiologists [De Visschere 2018]. Thus, the development of methods for accurate and automatic segmentation of the prostate on MRI scans is a significant and beneficial task in clinical applications.

Computer-aided methods for precise and automatic prostate segmentation present several clinical benefits, such as increased time efficiency, reduced errors, reproducibility, and invariance among radiologists [Ye et al. 2016]. Nevertheless, automatic prostate segmentation is a challenging task because of the wide variation in prostate anatomy due to pathological changes, tissue similar to the adjacent organs, and different acquisition protocols for MRI scans [De Visschere 2018].

Thereby, this paper proposes an automatic method for prostate segmentation on 3D MRI scans using a content-sensitive superpixels technique, phylogenetic indexes, YOLO convolutional network, XGBoost, and the particle swarm optimization (PSO) algorithm. As for contributions to the computer science field, we can mention: 1) detect the prostate region based on the YOLO convolutional network to decrease the region of interest, reducing the imbalance of the prostate and non-prostate classes, 2) describe the local prostate and non-prostate tissues using the phylogenetic indexes based on the IMSLIC algorithm and 3) build an XGBoost in conjunction with the PSO algorithm to distinguish prostate and non-prostate tissues.

In addition to Section 1, the paper is organized as follows. Section 2 describes some related works for prostate segmentation on 3D MRI scans. Section 3 details the materials and proposed method. Section 4 presents and discusses the results obtained. Finally, Section 5 presents the concluding observations and future works.

\section{Related works}

Accurate prostate segmentation in 3D MRI scans is very useful for cancer detection, biopsy, staging, monitoring, and treatment. In this regard, there is a huge demand for fast and accurate methods for clinical applications. In the automatic prostate segmentation task on MRI scans, [Jensen et al. 2019] described a method based on the Unet architecture obtaining a DSC of 74.3\%. [Wang et al. 2019] reported a method using a fully convolutional network (FCN) with a group dilated convolution presenting a DSC of $88 \pm 0.05 \%$. [Chen et al. 2020] proposed a method based on image-level labels resulting in a DSC of $83.39 \%$ and [Comelli et al. 2021] developed a method using the efficient neural network (ENet) obtaining a DSC of 90.8\%. Despite the various methods that have been proposed and published in recent literature with reasonable results, accurate and automatic prostate segmentation on MRI scans remains a difficult task. Since the methods present several challenges that can result in poor segmentation. For example, deep learning-based methods need a large amount of data, resulting in a slow training process. 


\section{Materials and method}

This section presents the proposed method for automatic prostate segmentation on 3D MRI scans. In addition to the material step, the proposed method contains three other steps: 2) preprocessing, 3) prostate detection, and finally, 4) prostate segmentation. Figure 1 illustrates the flowchart of the proposed method.

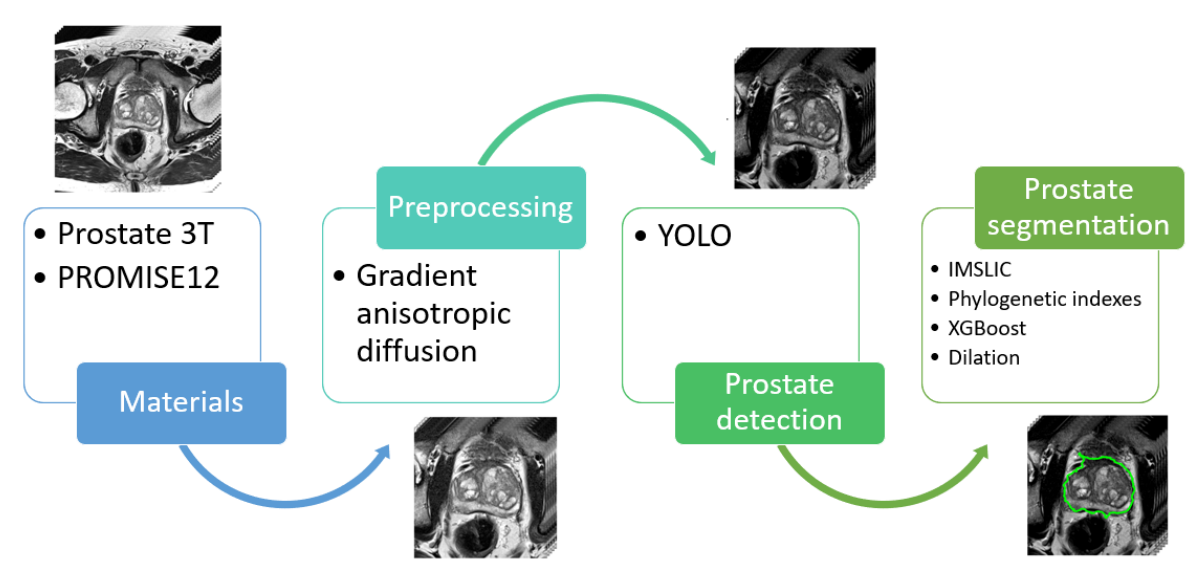

Figura 1. Proposed method for prostate segmentation on 3D MRI scans.

\subsection{Materials}

In order to validate the proposed method in complex situations, two different MRI databases are used together in the evaluation of the method. The Prostate 3T [Litjens et al. 2015] and PROMISE12 [Litjens et al. 2014] databases are available on the internet for challenges purpose. Both databases have a certain complexity since they have different aspects, such as spatial resolution, voxel spacing, the intensity of the magnetic field, dynamic range, and anatomic appearance into MRI scans [Litjens et al. 2014, Litjens et al. 2015].

In total, both databases contain $80 \mathrm{~T} 2$-weighted MRI scans along with their ground truth. Notwithstanding, 24 MRI scans from the PROMISE12 database that were acquired using an endorectal coil (ERC) were not used in the proposed method for several reasons. First, the coil deforms the prostate anatomy compressing the peripheral zone. Second, the gel used in the acquisition creates a regional T2 signal that can obscure the detection of cancer [De Visschere 2018]. Therefore, 56 MRI scans are used in the method.

\subsection{Preprocessing}

All 56 MRI scans were submitted to the preprocessing step. The objective of this step is to correct noises from the acquisition process, preserving the edges of the prostate. For this, the anisotropic diffusion filter using the modified curvature diffusion equation (MCDE) was applied [Johnson et al. 2015]. Qualitatively, MCDE compares well with other nonlinear diffusion filters. It is less sensitive to contrast than classic Perona-Malik style diffusion and preserves finer detailed structures in images, such as the prostate border in MRI scans. 


\subsection{Prostate detection}

The prostate detection step used the YOLO convolutional neural network [Redmon and Farhadi 2018]. This step is important, as it limits the scope of MRI scans to the prostate region, eliminating other unwanted details from the image, such as the other organs present in the image. The YOLO presents several benefits over the traditional techniques of object detection: 1) YOLO is extremely fast in detection, 2) uses information from the entire image to predict each bounding box, and finally, 3) has high power of generalization [Redmon and Farhadi 2018].

Due to the wide variation in size and shape between the different patients ascribed to pathological changes and different acquisition protocols, the following steps are applied slice by slice from the axial view. Figure 2 presents the flowchart of the prostate detection step. First, all MRI slices are resized to $256 \times 256$. Then, the prediction of the prostate regions is performed by YOLO. Third, find out the $x_{\min }, y_{\min }, x_{\max }$ and $y_{\max }$ coordinates among all bounding boxes of the same MRI scan. Finally, reconstruction of the 3D bounding box in the MRI scan using the coordinates preserving the $z$ axis.

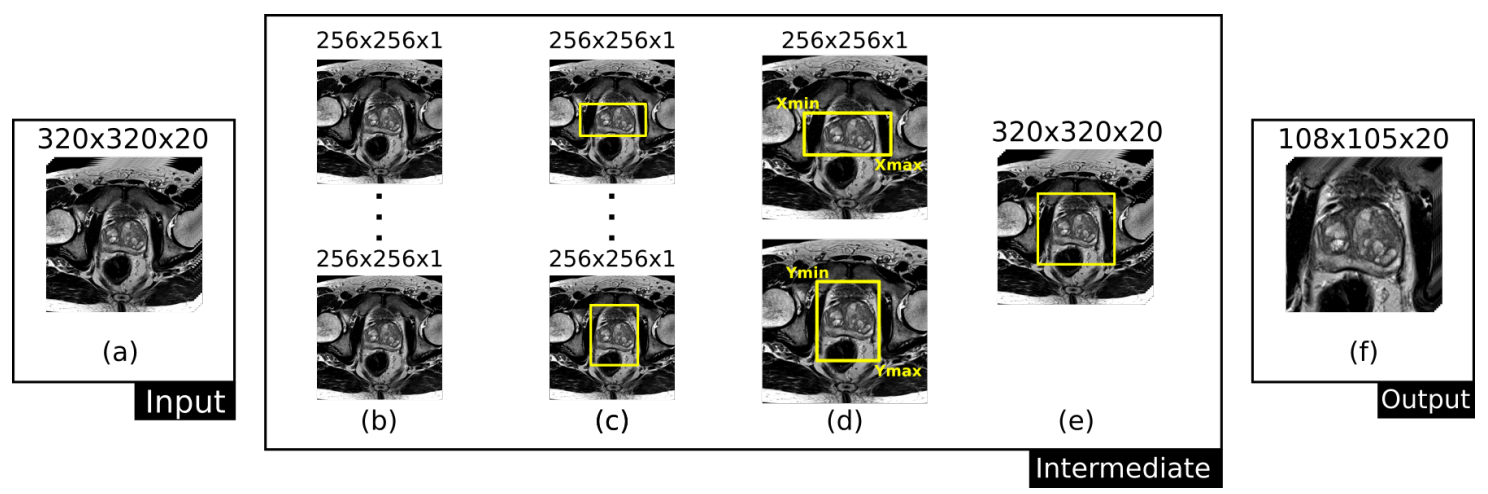

Figura 2. Flowchart of the prostate detection step.

\subsection{Prostate segmentation}

The prostate segmentation step combines a content-sensitive superpixels technique, texture features based on the phylogenetic indexes, an XGBoost in conjunction with the PSO algorithm, and a morphological operation for extracting the prostate surface. Thus, this step consists of four substeps: 1) superpixels clustering, 2) features extraction, 3) superpixels classification, and finally, 4) fine segmentation.

\subsubsection{Superpixels clustering}

The first substep consists of clustering the pixels into superpixels using the intrinsic manifold simple linear iterative clustering (IMSLIC) algorithm [Liu et al. 2017]. A superpixels-based analysis reduces the computational cost while potentially increasing the detection accuracy, as it is more robust to noise than pixels-based analysis. The IMSLIC algorithm presents advantages over the traditional superpixels techniques: 1) IMSLIC computes content-sensitive superpixels, 2) it can effectively capture nonhomogenous features, 3) has a good initialization of cluster centers, and 4) it presents few parameters [Liu et al. 2017]. Finally, each superpixel is centered on a 64 x 64 patch image. Figure 3 illustrates the application of the IMSLIC algorithm in an MRI scan. 


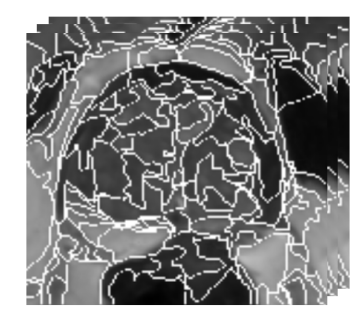

Figura 3. Application of the IMSLIC algorithm in an MRI scan.

\subsubsection{Features extraction}

The second substep consists of extracting texture features from superpixels using the phylogenetic indexes [Silva et al. 2016]. These indexes are based on diversity and the phylogenetic distance, accounting for the number of edges in a specific tree architecture. According to [Magurran 2005], diversity describes the variety of species present in a community or area. In order to use these concepts to describe prostate and non-prostate tissues, it is necessary to map the biologic concepts to MRI slice, as shown in Table 1.

Tabela 1. Mapping biologic concepts to MRI slice.

\begin{tabular}{cc}
\hline Biology & Prostate segmentation step \\
\hline Community & MRI slice \\
Species & Intensity values of the MRI slice \\
Species richness & Number of distinct voxels in a superpixel \\
Individual & Number of voxels of a particular species contained in a superpixel \\
\hline
\end{tabular}

There are several reasons to use the phylogenetic indexes for describing the local prostate and non-prostate tissues in this work: 1) MRI scans have a dynamic bit depth [Ghose et al. 2012], 2) both MRI databases were acquired using different protocols and equipment, presenting a greater diversity with different intensity values, and 3) these indexes have high potential in texture analysis in various medical applications [Silva et al. 2016, Costa et al. 2018, Cruz et al. 2020]. In total, 12 indexes were used, which are described below.

The relationship between two species randomly chosen in a phylogenetic tree of a community is presented by the taxonomic diversity $(\Delta)$ and taxonomic distinction $\left(\Delta^{*}\right)$ indexes [Clarke and Warwick 1998]. These taxonomic indexes are based on the number of species, the number of individuals, and the phylogenetic distance, that is, the number of edges in the phylogenetic tree [Clarke and Warwick 1998].

The taxonomic diversity $(\Delta)$ index computes the mean taxonomic distance between two individuals randomly chosen in a community [Clarke and Warwick 1998], namely:

$$
\Delta=\frac{\sum \sum_{i<j} w_{i j} x_{i} x_{j}}{[n(n-1) / 2]} .
$$

The taxonomic distinction $\left(\Delta^{*}\right)$ index calculates the mean taxonomic distance between two individuals of different species [Clarke and Warwick 1998], and it can be computed by:

$$
\Delta^{*}=\frac{\sum \sum_{i<j} w_{i j} x_{i} x_{j}}{\sum \sum_{i<j} x_{i} x_{j}}
$$


where $x_{i}(i=0, \ldots, s)$ and $x_{j}(j=0, \ldots, s)$ are the number of individuals of the $i$ and $j$ species, $s$ is the species richness, $n$ is the total number of individuals and $w_{i j}$ is the phylogenetic distance between the species $i$ and $j$ in a tree.

The intensive quadratic entropy $(J)$ index computes the mean phylogenetic distance between two randomly chosen species [Izsák and Papp 2000]:

$$
J=\left[\sum w_{i, j}\right] / s^{2} .
$$

The extensive quadratic entropy $(F)$ index calculates the sum of all pairwise phylogenetic distance [Izsák and Papp 2000], computed by:

$$
F=\sum w_{i, j}
$$

The average taxonomic distinctness $(A v T D)$ index computes the mean path length connecting species in a community [Clarke and Warwick 1998], and it is defined by:

$$
\left.A v T D=\left[\sum \sum_{i<j} w_{i j}\right] /[s(s-1)) / 2\right] .
$$

The total taxonomic distinctness (TTD) index calculates the mean phylogenetic distinctivenes summed over all species [Clarke and Warwick 1998], namely:

$$
T T D=\sum_{i}\left[\left(\sum_{j \neq i} w_{i j}\right) /(s-1)\right],
$$

where $w_{i, j}$ represents the phylogenetic distance between the species $i$ and $j$ in a tree and $s$ represents the number of species.

The pure diversity $\left(D_{D}\right)$ index computes the phylogenetic distance of a particular species to its nearest neighbor [Weitzman 1992], and is given by:

$$
D_{D}=\sum d_{i \min }
$$

where $d_{i \text { min }}$ indicates the phylogenetic distance from the nearest neighbour of the species $i$ to all other species.

The phylogenetic diversity $\left(P D_{N O D E}\right)$ index calculates the minimum total length of all phylogenetic branches necessary to measure a taxon in a phylogenetic tree and it can be calculated by:

$$
P D_{N O D E}=\sum n_{i}
$$

The $\left(P D_{R O O T}\right)$ index computes the number of nodes within the rooted (maximum) spanning path:

$$
P D_{R O O T}=\sum n_{\text {iROOT }}
$$

The average phylogenetic diversity $(A v P D)$ index calculates the mean phylogenetic diversity in a community:

$$
A v P D=P D_{N O D E} / s,
$$


where $n_{i}$ represents the number of $i$ nodes in the minimum path of each species present in the diversity, $n_{i R O O T}$ is the number of nodes in the path and $s$ represents the number of species.

In addition to these indexes, the $Q$ and $W$ indexes proposed by [Vane-Wright et al. 1991] were used to calculate the sum of the contributions of each species to diversity and the sum of standardized taxic weights, respectively.

\subsubsection{Superpixels classification}

The third substep consists of the superpixels classification in prostate and non-prostate tissues using an XGBoost [Chen and Guestrin 2016] in conjunction with the PSO algorithm [Rini et al. 2011]. Thus, it is necessary to label each superpixel in the prostate and non-prostate classes based on the ground truth. A superpixel is considered a prostate tissue if it has at least $70 \%$ of its pixels found in the ground truth. If a superpixel touches the ground truth in a proportion less than $70 \%$, it is ignored from the XGBoost training. Finally, all the others are labeled as non-prostate tissues.

The XGBoost algorithm has several advantages over traditional classification techniques: 1) XGBoost incorporates the concept of regularization to avoid overfitting, 2) it utilizes the power of parallel processing and cache optimization to speed up the training process, and 3) it employs the distributed weighted Quantile Sketch algorithm to effectively find the optimal split points among weighted databases [Chen and Guestrin 2016]. These advantages are ideal in the superpixels classification since the prostate and non-prostate tissues present class imbalance [Ghose et al. 2012].

Table 2 shows the particle coding of the XGBoost algorithm in conjunction with the PSO algorithm. Each particle has the following coordinates: $x_{1}$ represents the maximum depth of the tree with values between 1 and 10, $x_{2}$ represents the subsample ratio of columns when constructing each tree with values between 0 and $1, x_{3}$ represents the minimum sum of weights of all observations required in a child with values between 1 and $10, x_{4}$ represents the minimum loss reduction necessary to make a further partition on a leaf node of the tree with values between 0 and 1 , and finally, $x_{5}$ represents the learning rate with values between 0 and 1 .

Tabela 2. Particle definition in the superpixels classification substep.

\begin{tabular}{ccc}
\hline Particle coordinate & XGBoost parameter & Range \\
\hline$x_{1}$ & Maximum depth of a tree & 1 up to 10 \\
$x_{2}$ & Subsample ratio of columns & 0 up to 1 \\
$x_{3}$ & Minimum sum of weights of all observations & 1 up to 10 \\
$x_{4}$ & Minimum loss reduction & 0 up to 1 \\
$x_{5}$ & Learning rate & 0 up to 1 \\
\hline
\end{tabular}

Every single particle of the initial swarm is randomly initialized based on its range, as described in Table 2. The fitness of each particle is evaluated through the results obtained by the XGBoost algorithm on the validation subset using the selected parameters, as described in Equation 11. Once the stop criterion is satisfied, the particle represented by Gbest is the set of optimal parameters for the superpixels classification in prostate and non-prostate tissues. 


$$
\text { Fitness }=(2 * \text { Recall })+\text { Specificity }+ \text { Accuracy }
$$

The metric recall has been given a higher weight because it represents the model ability to correctly classify prostate tissue.

\subsubsection{Fine segmentation}

The last substep consists of smoothing the prostate contour using a morphological operation. The image reconstructed from the superpixels classification substep may present an irregular boundary when the superpixels do not find the prostate edge accurately. Therefore, the prostate surface should be refined to overcome this issue. In this substep, the dilation morphological operation [Gonzales and Woods 2002] with a $5 \mathrm{x}$ 5 circular structuring element is used in each MRI slice. Finally, the final prostate surface is generated for comparison with the ground truth.

\section{Results and discussion}

The Prostate 3T and PROMISE12 databases were randomly split into three subsets: training, validation, and test with the respective proportions $60 \%, 20 \%$, and $20 \%$. The training subset has the largest number of MRI scans to make the proposed method more robust and generic. In addition, the split process ensures a balanced number of MRI scans from both databases in each subset. Hence, the training subset has 34 MRI scans, the validation subset has $11 \mathrm{MRI}$ scans, and the test subset has $11 \mathrm{MRI}$ scans.

\subsection{Prostate detection results}

The prostate detection step was applied slice by slice from the axial view using the YOLO network. So, the training subset contains 471 slices, the validation subset contains 224 slices and the test subset contains 228 slices. The training of the network was defined for 150 epochs and the weights update was based on the Adam algorithm with a learning rate of 0.001 .

Table 3 presents the results in the prostate detection step on the validation and test subsets. The results include the mean intersection over union (IoU), recall (REC), precision (PREC), and the FN rate that represents the total of the prostate MRI slices that were not detected [Taha and Hanbury 2015]. In both subsets, the YOLO obtained a satisfactory result in detecting the prostate, presenting a low loss value. The total execution time of the prostate detection step was approximately 40 minutes.

Tabela 3. Results obtained in the prostate detection step on the validation and test subsets.

\begin{tabular}{cccccc}
\hline Subset & MRI slices & IoU (\%) & REC (\%) & PREC (\%) & FN \\
\hline Validation & 224 & 71.01 & 99.29 & 80.81 & 1 \\
Test & 228 & 71.85 & 97.63 & 87.77 & 4 \\
\hline
\end{tabular}




\subsection{Prostate segmentation results}

The prostate segmentation step, described in Section 3.4, detailed a conventional approach based on the IMSLIC algorithm, phylogenetic indexes, and the XGBoost algorithm with the PSO algorithm. All the texture features were submitted to the XGBoost model in conjunction with the PSO algorithm to distinguish prostate and non-prostate tissues. The XGBoost training used as objective function the binary logistic regression with a fixed number of 1,000 decision trees and the $\mathrm{L} 2$ regularization.

Table 4 presents the results obtained in both validation and test subsets for the Gbest particle at the end of the PSO algorithm. The results include mean recall (REC), mean specificity (SPE), and mean accuracy (ACC). The Gbest particle consists of the following coordinates: $x_{1}$ equals $9, x_{2}$ equals $0.852, x_{3}$ equals $6, x_{4}$ equals 0.480 , and finally, $x_{5}$ equals 1 . The particle coordinates have been seen in Table 2 . The metric recall that represents the correctly classified prostate superpixels showed higher values than the others, due to the ponderation attributed to the fitness of the particle. The total execution time of the optimization of the XGBoost algorithm parameters was approximately 8 hours.

Tabela 4. Results obtained by the Gbest particle on the validation and test subsets.

\begin{tabular}{ccccc}
\hline Subset & Superpixels & REC (\%) & SPE (\%) & ACC (\%) \\
\hline Validation & 4,852 & 99.73 & 96.72 & 99.39 \\
Test & 15,698 & 95.81 & 85.65 & 93.90 \\
\hline
\end{tabular}

Table 5 presents the results obtained in the prostate segmentation step on the test subset (11 MRI scans). The results include the dice similarity coefficient (DSC), relative volume difference (RVD), volumetric similarity (VS), recall (REC), specificity (SPE), and accuracy (ACC) [Taha and Hanbury 2015].

Tabela 5. Results obtained in the prostate segmentation step on the test subset.

\begin{tabular}{ccccccc}
\hline Statistic & DSC (\%) & RVD (\%) & VS (\%) & REC (\%) & SPE (\%) & ACC (\%) \\
\hline Min & 79.86 & 5.40 & 97.36 & 82.02 & 90.14 & 87.74 \\
Max & 89.70 & 6.46 & 96.86 & 92.60 & 91.82 & 92.11 \\
Mean & $\mathbf{8 4 . 4 8}$ & $\mathbf{8 . 7 6}$ & $\mathbf{9 5 . 9 1}$ & $\mathbf{8 8 . 1 4}$ & $\mathbf{9 1 . 3 1}$ & $\mathbf{9 0 . 4 0}$ \\
Std. deviation & $\mathbf{3 . 1 0}$ & $\mathbf{8 . 6 8}$ & $\mathbf{3 . 8 1}$ & $\mathbf{3 . 9 0}$ & $\mathbf{2 . 2 1}$ & $\mathbf{1 . 4 9}$ \\
\hline
\end{tabular}

Experimental results shown in Table 5 demonstrate the satisfactory performance of the proposed method for prostate segmentation on 3D MRI scans. The method obtained a mean DSC of $84.48 \%$ with a standard deviation of $3.10 \%$, minimum DSC of $79.86 \%$, and a maximum DSC of $89.70 \%$. In addition, a low and positive mean RVD of $8.76 \%$ indicates that the final prostate surface is close to the ground truth, being slightly larger than the region performed manually by the radiologist. Finally, the mean of the metrics of VS, REC, SPE, and ACC present good precision of the voxels classified correctly.

\subsection{Case study}

In order to evaluate the qualitative results of the proposed method on the test subset, two MRI scans were selected based on the smallest and largest DSC presented in Table 5. 
Figure 4 (a) presents the result obtained with $89.70 \%$ of DSC and Figure 4 (b) presenting a DSC of $79.86 \%$. In both images, the final segmentation is highlighted in yellow and the ground truth is outlined in red.

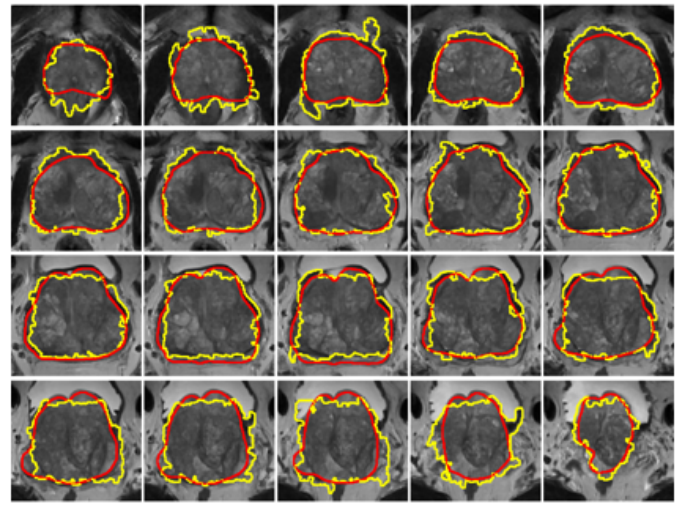

(a)
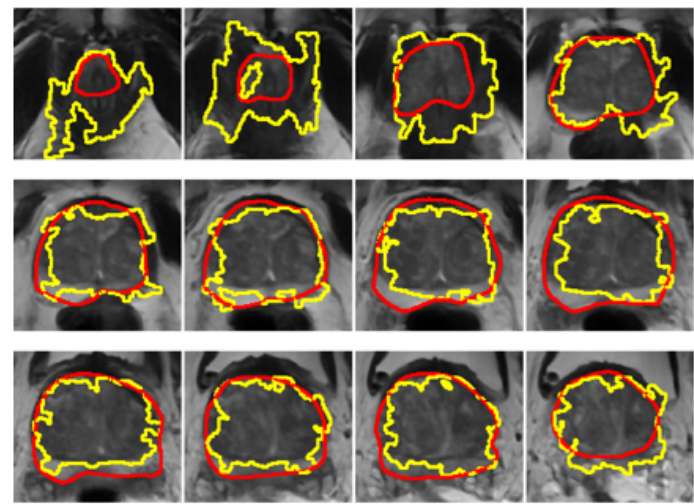

(b)

Figura 4. Case study. (a) Success case and (b) fail case.

In summary, most MRI slices had the final segmentation (yellow outline) similar to the ground truth (red outline). Generally, errors in prostate segmentation occur in the basal and apical regions, since the regions present different anatomy from the middle region. Besides, these regions present transitions with other organs, the basal region continues in the neck of the bladder, and the apical region is located on the superior surface of the urogenital diaphragm [Lee et al. 2011].

\section{Conclusion}

This paper proposed an automatic method for segmenting the prostate on 3D MRI scans based on the IMSLIC superpixel technique, texture analysis using phylogenetic indexes, and the XGBoost algorithm in conjunction with the PSO algorithm. In addition, the proposed method included the prostate detection step using the YOLO convolutional network. The results obtained demonstrate the high-performance potential of the method in two different MRI databases.

Future works include adding information from the neighborhood in the superpixels classification substep, thus avoiding flaws in the internal regions of the prostate. Besides, the use of more powerful techniques as an active contour for the refinement of the prostate surface, since the texture analysis with the phylogenetic indexes and the XGBoost algorithm showed satisfactory results in distinguishing between the prostate and non-prostate tissues. Finally, study image enhancement techniques to include the MRI scans acquired using an endorectal coil.

\section{Acknowledgements}

The authors acknowledge CAPES and CNPq for their financial support.

\section{Referências}

ACS (2021). American cancer society: Key statistics for prostate cancer. https://www.cancer.org/cancer/prostate-cancer/about/ key-statistics.html. Accessed: 2021-03-14. 
Chen, T. and Guestrin, C. (2016). Xgboost: A scalable tree boosting system. In Proceedings of the 22nd acm sigkdd international conference on knowledge discovery and data mining, pages 785-794.

Chen, Z., Tian, Z., Li, X., Zhang, Y., and Dormer, J. D. (2020). Exploiting confident information for weakly supervised prostate segmentation based on image-level labels. In Medical Imaging 2020: Image-Guided Procedures, Robotic Interventions, and Modeling, volume 11315, page 1131523. International Society for Optics and Photonics.

Clarke, K. and Warwick, R. (1998). A taxonomic distinctness index and its statistical properties. Journal of applied ecology, 35(4):523-531.

Comelli, A., Dahiya, N., Stefano, A., Vernuccio, F., Portoghese, M., Cutaia, G., Bruno, A., Salvaggio, G., and Yezzi, A. (2021). Deep learning-based methods for prostate segmentation in magnetic resonance imaging. Applied Sciences, 11(2):782.

Costa, R. W. d. S., da Silva, G. L. F., de Carvalho Filho, A. O., Silva, A. C., de Paiva, A. C., and Gattass, M. (2018). Classification of malignant and benign lung nodules using taxonomic diversity index and phylogenetic distance. Medical \& biological engineering \& computing, 56(11):2125-2136.

Cruz, L. B. d., Souza, J. C., de Sousa, J. A., Santos, A. M., de Paiva, A. C., de Almeida, J. D. S., Silva, A. C., Junior, G. B., and Gattass, M. (2020). Interferometer eye image classification for dry eye categorization using phylogenetic diversity indexes for texture analysis. Computer Methods and Programs in Biomedicine, 188:105269.

De Visschere, P. (2018). Improving the diagnosis of clinically significant prostate cancer with magnetic resonance imaging. Journal of the Belgian Society of Radiology, 102(1).

Ghose, S., Oliver, A., Martí, R., Lladó, X., Vilanova, J. C., Freixenet, J., Mitra, J., Sidibé, D., and Meriaudeau, F. (2012). A survey of prostate segmentation methodologies in ultrasound, magnetic resonance and computed tomography images. Computer methods and programs in biomedicine, 108(1):262-287.

Gonzales, R. C. and Woods, R. E. (2002). Digital image processing.

INCA (2021). Instituto nacional do câncer, tipos de câncer: Próstata. http://www2.inca.gov.br/wps/wcm/connect/tiposdecancer/ site/home/prostata. Accessed: 2021-03-14.

Izsák, J. and Papp, L. (2000). A link between ecological diversity indices and measures of biodiversity. Ecological Modelling, 130(1-3):151-156.

Jensen, C., Sørensen, K. S., Jørgensen, C. K., Nielsen, C. W., Høy, P. C., Langkilde, N. C., and Østergaard, L. R. (2019). Prostate zonal segmentation in $1.5 \mathrm{t}$ and $3 \mathrm{t} \mathrm{t} 2 \mathrm{w}$ mri using a convolutional neural network. Journal of Medical Imaging, 6(1):014501.

Johnson, H. J., McCormick, M. M., and Ibanez, L. (2015). The itk software guide book 1: Introduction and development guidelines fourth edition updated for itk version 4.7. Kitware Inc.

Lee, C. H., Akin-Olugbade, O., and Kirschenbaum, A. (2011). Overview of prostate anatomy, histology, and pathology. Endocrinology and Metabolism Clinics, 40(3):565-575. 
Litjens, G., Futterer, J., and Huisman, H. (2015). Data from prostate-3t: The cancer imaging archive. http://doi.org/10.7937/K9/TCIA.2015.QJTV5IL5. Accessed: 2020-03-15.

Litjens, G., Toth, R., van de Ven, W., Hoeks, C., Kerkstra, S., van Ginneken, B., Vincent, G., Guillard, G., Birbeck, N., Zhang, J., et al. (2014). Evaluation of prostate segmentation algorithms for mri: the promise12 challenge. Medical image analysis, 18(2):359-373.

Liu, Y.-J., Yu, M., Li, B.-J., and He, Y. (2017). Intrinsic manifold slic: a simple and efficient method for computing content-sensitive superpixels. IEEE transactions on pattern analysis and machine intelligence.

Magurran, A. E. (2005). Species abundance distributions: pattern or process? Functional Ecology, 19(1):177-181.

Paiva, A. M., Diniz, J. O., Silva, A., and Paiva, A. (2019). Segmentação de vértebras e diagnóstico de fraturas em imagens de ressonância magnética utilizando u-net $3 \mathrm{~d}$ e deep belief network. In Anais do XIX Simpósio Brasileiro de Computação Aplicada à Saúde, pages 106-117, Porto Alegre, RS, Brasil. SBC.

Redmon, J. and Farhadi, A. (2018). Yolov3: An incremental improvement. Computing Research Repository (CoRR).

Rini, D. P., Shamsuddin, S. M., and Yuhaniz, S. S. (2011). Particle swarm optimization: technique, system and challenges. International journal of computer applications, 14(1):19-26.

Siegel, R. L., Miller, K. D., and Jemal, A. (2019). Cancer statistics, 2019. CA: a cancer journal for clinicians, 69(1):7-34.

Silva, G. L. F. d., Carvalho Filho, A. O. d., Silva, A. C., Paiva, A. C. d., and Gattass, M. (2016). Taxonomic indexes for differentiating malignancy of lung nodules on ct images. Research on Biomedical Engineering, 32(3):263-272.

Taha, A. A. and Hanbury, A. (2015). Metrics for evaluating 3d medical image segmentation: analysis, selection, and tool. BMC medical imaging, 15(1):29.

Vane-Wright, R. I., Humphries, C. J., and Williams, P. H. (1991). What to protect?-systematics and the agony of choice. Biological conservation, 55(3):235254.

Wang, B., Lei, Y., Tian, S., Wang, T., Liu, Y., Patel, P., Jani, A. B., Mao, H., Curran, W. J., Liu, T., et al. (2019). Deeply supervised 3d fully convolutional networks with group dilated convolution for automatic mri prostate segmentation. Medical physics, 46(4):1707-1718.

Weitzman, M. L. (1992). On diversity. The Quarterly Journal of Economics, 107(2):363405.

Ye, X., Oyoyo, U., Lu, A., Dixon, J., Rojas, H., Randolph, S., and Kelly, T. (2016). Comparison of $3.0 \mathrm{t}$ pac versus $1.5 \mathrm{t}$ erc mri in detecting local prostate carcinoma. bioRxiv, page 058123 . 